\title{
Contrainte du doublage clitique et détachement de l'objet à gauche
}

\author{
Etienne Riou ${ }^{1,2}$, et Barbara Hemforth ${ }^{1}$ \\ ${ }^{1}$ LLF, Bât. Olympe de Gouges, 5ème étage. 8, Rue Albert Einstein 75013 Paris, France \\ ${ }^{2}$ FoReLL, Bât. A5. 5, rue Théodore Lefebvre 86073 Poitiers Cedex 9, France \\ etienne.riou@univ-poitiers.fr
}

Résumé. Dans cet article, nous abordons empiriquement les propriétés pragmatiques de deux constructions permettant le détachement de l'objet à gauche : la dislocation clitique à gauche et la topicalisation simple. Ces constructions se différencient l'une de l'autre par la présence ou non d'un pronom clitique reprenant le rôle de l'objet détaché dans la phrase racine. Bien qu'elles soient similaires pragmatiquement, nous faisons l'hypothèse que la topicalisation simple soit plus restreinte dans un contexte discursif contrastif. En dehors de ce contexte, le détachement à gauche de l'objet doit prendre un pronom clitique objet. Dans une approche à contraintes multiples, cette restriction est considérée comme une contrainte noncatégorique et peut être explorée en tant que telle via une tâche de jugements d'acceptabilité. Pour ce faire, nous utilisons le paradigme expérimental du «test de jugements d'acceptabilité accélérés». Les résultats montrent que la violation de cette contrainte des topicalisation de l'objet conduit à un niveau d'acceptabilité caractéristique des contraintes non-catégoriques : plus haut qu'une construction agrammaticale mais plus bas qu'une construction bien formée dans un contexte approprié.

\begin{abstract}
Clitic doubling constraint and object left detachment. In this paper, we empirically investigate the pragmatic properties of two constructions allowing the left-detachment of objects: clitic left dislocation and simple topicalization. These constructions can be differentiated by the presence or the absence of a clitic pronoun assuming the role of the detached object in the root sentence. Although they are pragmatically similar, we argue that simple topicalization is more restricted in a contrastive discursive context. Outside of this context, object left detachment must include an object clitic pronoun. In a constraint-based approach, this restriction is considered to be a non-categorical constraint and can be investigated as such with an acceptability judgments task. To do this, we apply the experimental paradigm of "speeded acceptability judgments". The results show that the violation of this constraint by object topicalization leads to an acceptability level typical of non-categorical constraint: higher than an ungrammatical construction but lower than a well-formed construction in an appropriate context.
\end{abstract}




\section{Le détachement à gauche}

Nous nous intéressons ici à deux formes de détachement à gauche : la dislocation à gauche et la topicalisation simple. Ces deux constructions sont distinctes syntaxiquement (Cinque, 1990), mais leurs emplois sont contraints par différentes propriétés pragmatiques (DelaisRoussarie et al, 2004). Cet article se focalise sur les variantes objet de ces deux constructions ainsi que sur l'effet des propriétés pragmatiques sur leur acceptabilité en contexte. Les deux sous-sections suivantes établissent les différentes propriétés du détachement de l'objet à gauche que nous abordons empiriquement.

Dans cette perspective, il est intéressant de noter que les détachements à gauche sont souvent considérés comme appartenant au français oral informel ou non-standard (Lambrecht, 1994 ; De Cat, 2007). Cela est susceptible d'influencer négativement la perception de ces constructions, notamment dans le cadre d'une expérience de jugements d'acceptabilité (Zribi-Hertz, 1994 ; Riou \& Hemforth, 2015). Notre approche devra prendre en compte ce facteur pour les deux constructions.

\subsection{La dislocation à gauche}

La dislocation à gauche est une construction dans laquelle un élément est détaché dans la périphérie gauche de la phrase racine et repris dans la phrase racine par un élément résomptif. Dans cet article, nous nous focaliserons sur le cas où l'élément résomptif est un pronom clitique (la dislocation clitique à gauche ou « $\mathrm{DCG} »)$. Ce phénomène est possible pour tous les constituants pronominalisables. Les exemples suivants (issus de DelaisRoussarie et al, (2004)) l'illustrent pour le sujet (1), le complément objet (2) et le complément oblique (3).

1. Mon frère, il va bien.

2. Cet homme, je ne le connais pas.

3. A ce confort, on s'y habitue très vite.

Cette construction est associée à une expression particulière de la structure informationnelle (Lambrecht, 1994 ; De Cat, 2007). La dislocation à gauche est marquée par la topicalisation de l'élément détaché à gauche et à la focalisation du prédicat (4). Dans cet exemple, le sujet détaché à gauche est ce dont la question est à propos (le topique), tandis que le prédicat correspond à la réponse à la question (le focus).

4. Q : Qu'est-ce qui est arrivé à ta voiture?

$\mathrm{R}$ : Ma voiture elle est en panne.

La dislocation à gauche se distingue ainsi d'autres constructions non-canoniques telles que les clivées qui sont caractérisées par une autre expression de la structure informationnelle. La clivée en «c'est» (5) est marquée par la focalisation du constituant clivé. La clivée en «j'ai» (6) est marquée par la focalisation de l'ensemble de la proposition.

5. Q : Il paraît que ta moto est en panne?

$\mathrm{R}$ : C'est ma voiture qui est en panne.

6. Q : Qu'est-ce qui s'est passé ?

$\mathrm{R}$ : J'ai ma voiture qui est en panne.

\subsection{La topicalisation simple}

Dans le cas de détachement des constituants non-sujet (exemples 2 et 3), il est possible de ne pas les reprendre dans la phrase racine. On a alors à faire à une «topicalisation » (Cinque, 1990), que nous appellerons ici «topicalisation simple», dans la mesure où la dislocation à gauche est également une forme de topicalisation. 
Les topicalisations simples sont également associées à la topicalisation du constituant disloqué, mais elles semblent également plus contraintes en termes de contexte discursif. Elles apparaissent notamment plus acceptables dans un contexte de topicalisation contrastive avec des alternatives mentionnées (Kerleroux et Marandin, 2001). Dans l'exemple (7), les compléments second («aux filles » et «aux garçons ») sont des sousensembles alternatifs de l'ensemble « les élèves ».

7. Marie a réuni les élèves. Aux filles, elle a donné des exercices d'algèbre. Aux garçons, elle a dicté un problème de géométrie.

Dans cet article, nous nous intéressons à l'acceptabilité de la variante objet de cette construction (8) ainsi que sa possible alternance avec la DCG (9).

8. Marie a distribué les exercices. Les exercices d'algèbres, (\#?) elle a donnés aux filles. Le problème de géométrie, elle a dicté aux garçons.

9. Marie a distribué les exercices. Les exercices d'algèbres, elle les a donnés aux filles. Le problème de géométrie, elle l'a dicté aux garçons.

Les topicalisations simples de $(8)$ sont des exemples très particuliers de topicalisations simples, car, malgré l'absence de pronom clitique, l'élément détaché à gauche est clairement marqué comme étant l'objet du verbe de la phrase racine. Cette relation est indiquée via le contexte discursif, mais aussi par l'accord du participe passé avec l'objet dans l'exemple (9). De ce point de vue, notre distinction «topicalisation/dislocation » dépend uniquement de la présence ou l'absence d'un pronom clitique (contrairement à Cinque (1990) qui inclut un ensemble de relations plus complexes entre l'élément disloqué et la DCG).

Nous faisons l'hypothèse que, contrairement à la dislocation de l'objet, la topicalisation simple de l'objet est acceptable dans des contextes contrastifs lorsqu'une alternative est mentionnée. En effet, dans les contextes des exemples (8) et (9), les deux constructions paraissent acceptables. Cependant, si l'on choisit de ne pas mentionner une alternative, la topicalisation simple (10) semble moins acceptable que la DCG (11).

10. Marie a distribué les exercices. \#Le problème de géométrie, elle a dicté aux garçons.

11. Marie a distribué les exercices. Le problème de géométrie, elle l'a dicté aux garçons.

Dans la suite, nous cherchons à tester cette intuition de manière empirique et quantitative. Pour ce faire, nous choisissons d'adopter une approche à contraintes distinguant des contraintes catégoriques et non-catégoriques.

\section{Approche à contraintes}

\subsection{Cadre général}

Cette approche postule que l'emploi d'une construction est conditionné par différents types de contraintes et que ces contraintes peuvent être explorées via la gradation de l'acceptabilité lorsqu'elles sont violées (Sorace et Keller, 2005). Les deux types de contraintes (catégoriques et non-catégoriques) se caractérisent par leurs effets sur l'acceptabilité.

Les contraintes catégoriques (ou contraintes «dures») et leurs violations ont un effet plus important sur l'acceptabilité d'une construction que la violation de contraintes noncatégoriques (ou contraintes «souples »). C'est-à-dire qu'une construction violant une contrainte non-catégoriques sera perçue comme plus acceptable qu'une construction violant une contrainte catégorique, mais moins acceptable qu'une construction bien formée et dans un contexte adapté. Cependant, la violation de plusieurs contraintes non-catégoriques peut avoir des effets additifs qui peuvent atteindre l'effet de la violation d'une contrainte 
catégorique. C'est-à-dire qu'une construction violant plusieurs contraintes non-catégoriques sera perçue aussi inacceptable qu'une construction ne violant qu'une seule contrainte catégorique.

Les contraintes catégoriques sont souvent associées aux contraintes morphosyntaxiques et les contraintes non-catégoriques aux contraintes liées à l'interface entre discours et syntaxe (telles que la structure informationnelle). Ce n'est cependant pas toujours le cas, car il existe des contraintes morphosyntaxiques non-catégoriques (Blache et al, 2006). Dans la suite de cet article, nous ne distinguerons que les contraintes catégoriques des contraintes non-catégoriques, sans les associer à des domaines linguistiques particuliers.

En ce qui concerne les détachements de l'objet à gauche, nous faisons l'hypothèse que les propriétés pragmatiques établies précédemment (topicalisation de l'objet disloqué et mention d'alternatives dans un contexte contrastif) correspondent à des contraintes noncatégoriques. Nous postulons donc que la violation d'une de ces contraintes conduirait à une baisse de l'acceptabilité par rapport à une construction bien formée et dans un contexte adapté, mais moins importante que dans le cas d'une construction agrammaticale. La contrainte non-catégorique qui nous intéresse a déjà été abordée dans la littérature sur les approches à contraintes de l'acceptabilité.

\subsection{La contrainte du doublage clitique}

La contrainte du doublage clitique des objets préverbaux fait partie d'un ensemble de contraintes non-catégoriques du détachement de l'objet à gauche qui ont notamment été explorées empiriquement en grec (Keller et Alexopoulou, 2001 ; Sorace et Keller, 2005). En grec, le doublage clitique est obligatoire pour l'objet préverbal et inacceptable pour le sujet préverbal. Les contraintes de l'ordre des constituants recoupent les contraintes informationnelles mentionnées précédemment (l'objet détaché à gauche doit être une information mentionnée ou inférable depuis le discours) ainsi que des contraintes prosodique (la position de l'accent focus sur le constituant le plus à droite).

Ces contraintes varient en fonction de leur effet sur l'acceptabilité. L'exemple (12) (Sorace et Keller, 2005: 310) contient un objet préverbal sans doublage clitique (les majuscules indiquent l'accent focus). Il est perçu plus acceptable qu'une phrase focalisant l'objet préverbal, mais moins acceptable qu'une phrase avec un doublage clitique et un sujet préverbal.

12. \#To Yani apelise i MARIA.

Le-Yanis-ACC a-viré la-Maria-NOM

Yanis, Maria a viré.

Le français diffère du grec, car l'ordre des mots y est moins libre. Il n'est pas possible pour un objet d'adopter une position préverbale et la position postverbale du sujet est plus restreinte. Elle n'est par exemple pas possible lorsque le verbe de la phrase est transitif (13). En outre, l'expression de la structure informationnelle tend à se faire de manière plus morphosyntaxique que prosodique (Lambrecht, 1994), même si la prosodie joue également un rôle (Beyssade et al, 2015).

13. *Les secrétaires a virés MARIE.

Le français est également caractérisé par une dépendance syntaxique importante entre les verbes et les clitiques. Ces derniers apparaissent souvent près du verbe même lorsqu'ils sont redondants (dans une coordination par exemple (14); Miller, 1992 : 158) et, peuvent exister sans antécédents, même dans le cas des clitiques objets (15).

14. Je frappe et i'entre.

15. La haine le dispute à l'envie.

Nous pensons tout de même que les contraintes en lien avec la structure informationnelle de l'objet préverbal en grec sont aussi valables pour le détachement de l'objet en français. Dans le cas des constructions à détachement, l'objet détaché doit être 
une information mentionnée ou inférable dans le discours, il ne peut prendre un accent focus (il peut en revanche prendre un accent contrastif; Marandin et al, 2002) et il est préféré avec un doublage clitique. Nous nous focalisons ici sur l'exploration empirique de cette dernière contrainte.

Cependant, nous adoptons une interprétation un peu différente. En effet, comme mentionnée dans la section précédente, nous faisons l'hypothèse que le contexte discursif joue un rôle quant à la perception de la contrainte de doublage clitique. Dans un contexte contrastif avec alternative mentionnée, il nous paraît possible que cette contrainte perde de son effet sur l'acceptabilité. En revanche, dans un contexte contrastif sans mention d'alternative, la contrainte est pleinement active. Notre objectif étant de distinguer les DCG des topicalisations simples, nous emploierons ce dernier contexte dans notre tâche de jugement d'acceptabilité.

De plus, nous prendrons en compte un autre type de contraintes qui n'est pas considéré dans (Keller et Alexopoulou, 2001). En effet, nous sommes susceptibles de rencontrer une difficulté liée au statut informel ou non-standard des détachements à gauche. Comme nous l'avons mentionné dans la section précédente, ce statut peut se traduire par une baisse de l'acceptabilité pour ces constructions. Dans le cas où cette baisse de l'acceptabilité soit équivalente à la violation d'une contrainte non-catégorique, il deviendrait alors difficile de distinguer les effets de contraintes non-catégoriques des effets de contraintes catégoriques. En effet, l'effet des contraintes non-catégoriques étant cumulatif, une construction affectée par son statut non-standard et la violation d'une contrainte non-catégorique serait perçue comme aussi inacceptable qu'une construction agrammaticale.

La méthodologie choisie doit pouvoir réduire l'effet de ce statut non-standard des détachements à gauche, en plus de pouvoir offrir la possibilité de graduer l'acceptabilité pour nos différentes conditions. C'est pourquoi nous avons choisi d'employer le paradigme du test de jugements d'acceptabilité accélérés.

\section{La méthodologie}

Le paradigme expérimental employé est celui des tests de jugements d'acceptabilité accélérés. Il s'inspire des expériences de jugements de Bader et Häussler (2010). Les participants sont exposés aux items et doivent indiquer leur jugement de manière binaire («acceptable » ou « inacceptable »). Contrairement aux tests de jugements d'acceptabilité standard, les participants ont un temps limité pour comprendre et juger les items.

Ce temps limité force les participants à se concentrer sur la lecture et la compréhension des différents items présentés. De plus, le caractère immédiat du jugement les aide à s'appuyer principalement sur leurs premières intuitions pour établir leur jugement d'acceptabilité. L'adoption d'un jugement binaire à la place d'un jugement gradué de 1 à 10 a deux avantages. Il permet d'accélérer la prise de jugement et fournit ainsi des temps de réaction qui donnent des informations supplémentaires quant au traitement de l'item par les participants. Les participants ont moins de temps pour choisir la note à donner. La gradualité du jugement peut être retrouvée à travers le calcul des proportions de jugements « acceptable » et « inacceptable » pour chaque condition.

Ce paradigme a également l'avantage d'être moins sensible aux éventuels effets du statut informel ou non-standard de certaines conditions. Nous faisons l'hypothèse que le temps limité dont les participants disposent permet de réduire l'effet des jugements métalinguistiques liés au statut non-standard des détachements. Cette contrainte est similaire à celle qui pousse les locuteurs à produire et comprendre sans difficulté les détachements à gauche dans le discours oral spontané. 


\section{L’expérience}

\subsection{Protocole et matériel}

En termes de protocole, le test de jugements d'acceptabilité accélérés permet de fixer exactement le temps durant lequel les participants ont un temps limité pour appréhender les items et répondre au test. En effet, les mots sont présentés un par un au centre de l'écran. Chacun d'entre eux reste visible pendant une durée de base de $225 \mathrm{~ms}$, plus $25 \mathrm{~ms}$ par caractère. Ils disparaissent ensuite pour faire place aux mots suivants jusqu'à la fin de l'item. Le participant a ensuite jusqu'à deux secondes pour effectuer un jugement binaire (« acceptable » ou « inacceptable ») sur l'item qu'il vient de lire. S'il est trop lent, le test ne prend pas en compte sa réponse et passe automatiquement à l'item suivant.

Le matériel croise les détachements à gauche (ici annotés « détachement » par soucis de brièveté) et les constructions clivées ( « clivée ») avec la présence et l'absence d'un pronom clitique reprenant le constituant disloqué ou clivé. L'exemple (16) illustre un item complet.

16. Q $[\mathrm{DG}]$ : La marine emprunte-t-elle des navires ?

$\mathrm{R}$ [détachement ; + clitique] : Oui. Les frégates, elle les emprunte au pays voisin.

$\mathrm{R}$ [détachement ; - clitique] : \# Oui. Les frégates, elle emprunte au pays voisin.

$\mathrm{Q}$ [clivée] : La marine emprunte bien les porte-avions?

$\mathrm{R}$ [clivée ; + clitique] : *Non. Ce sont les frégates qu'elle les emprunte.

$\mathrm{R}$ [clivée ; - clitique] : Non. Ce sont les frégates qu'elle emprunte.

Les clivées comme les détachements à gauche sont présentés dans un contexte discursif adapté (Brunetti et al, 2012 ; Hamlaoui, 2009) : la topicalisation contrastive pour les DG (entre l'ensemble « des navires » et le sous-ensemble « les frégates » dans l'exemple) et la focalisation corrective pour les clivées (entre «les porte-avions» et «les frégates» dans l'exemple).

Le croisement de ces constructions avec la présence ou l'absence de clitique a un effet différent selon la construction. Le détachement sans clitique (topicalisation simple) reste grammatical. Il est cependant dans un contexte discursif peu approprié, car on attend la mention d'une autre alternative (similaire à l'exemple de la section précédente). La clivée avec clitique est agrammaticale. Les DCG avec clitique et les clivées sans clitique sont grammaticales et dans des contextes appropriés.

Chaque item contient entre 12 mots (au minimum) et 16 mots (au maximum). L'ensemble de l'item (question et réponse) est présenté de manière accélérée. Nous avons évité l'emploi des pronoms clitiques «l' », «le », «la » et employé uniquement le pronom « les ", car son nombre de caractères ne varie pas et nous évitons des phénomènes de contraction avec le verbe («les emprunte » au lieu de «l'emprunte » dans l'exemple (16)) qui compliquent la présentation mot à mot.

Pour cette expérience, nous ${ }^{1}$ avons employé le logiciel E-prime pour appliquer notre protocole. E-prime (Schneider et al, 2012ab) est un logiciel permettant de créer des expériences psycholinguistiques variées avec un enregistrement précis des temps de réaction. Nos $30^{2}$ items ont ensuite été mélangés avec 30 distracteurs. Nous avons également inclus 2 conditions agrammaticales en plus à chacun de nos items pour avoir autant d'items grammaticaux que d'items agrammaticaux. Dans le cadre de cet article, ces conditions supplémentaires sont considérées comme des distracteurs. L'exemple (17) indique les conditions supplémentaires de l'item illustré dans l'exemple (16).

17. Q (supplémentaire 1) : Est-ce que la marine emprunter des navires ?

$\mathrm{R}$ (supplémentaire 1) : Oui. Je pense que tu as raison.

Q (supplémentaire 2): Est-ce que la marine emprunte des navires?

$\mathrm{R}$ (supplémentaire 2) : Oui. Je penser que tu as raison.

Le tout a été intégré au logiciel sous la forme de six listes avec une distribution des items par liste suivant le carré latin. Les six listes contiennent toutes les conditions avec une 
condition différente de chaque item par liste et le même nombre d'items par condition. Elles comprennent aussi le nombre de caractères de chaque mot (18) et sa durée de présentation (19).

18. marine $->6$

19. $6->225+25 * 6->375 \mathrm{~ms}$

Lorsqu'un participant passe l'expérience, E-prime choisit une liste selon l'ordre de passage. Le premier participant voit la première liste, le deuxième participant voit la deuxième liste, etc. Lorsque les six listes sont déjà passées une fois, E-prime repasse à la première. Le septième participant voit donc la première liste, le huitième voit la deuxième liste, etc.

Chaque item de la liste choisie est ensuite présenté une seule fois dans un ordre aléatoire. Lorsque tous les mots de l'item ont été présentés (question et réponse), le participant a jusqu'à deux secondes pour indiquer son jugement en appuyant sur la touche du clavier correspondante ( « $\mathrm{s}$ » pour « acceptable » et « $1 »$ pour « inacceptable »). E-prime enregistre alors sa réponse ainsi que son temps de réaction à partir du moment où le jugement est demandé. Pour éviter une fatigue naturelle due à la nature accélérée de l'expérience, le participant peut prendre une pause entre deux items. Quand tous les items de la liste (items cibles et distracteurs) ont été présentés, l'expérience est achevée.

\subsection{Participants}

Les participants ont été recrutés via la plate-forme du RISC et ils ont passé l'expérience dans une pièce insonorisée sur un ordinateur portable. Le test a été passé par 33 sujets francophones natifs de 18 à 75 ans (âge moyen : 34,5). Chacun des participants est venu sur place et a passé l'expérience dans une cabine insonorisée contre un dédommagement de $5 €$.

\subsection{Résultats}

\subsubsection{Jugements d'acceptabilité}
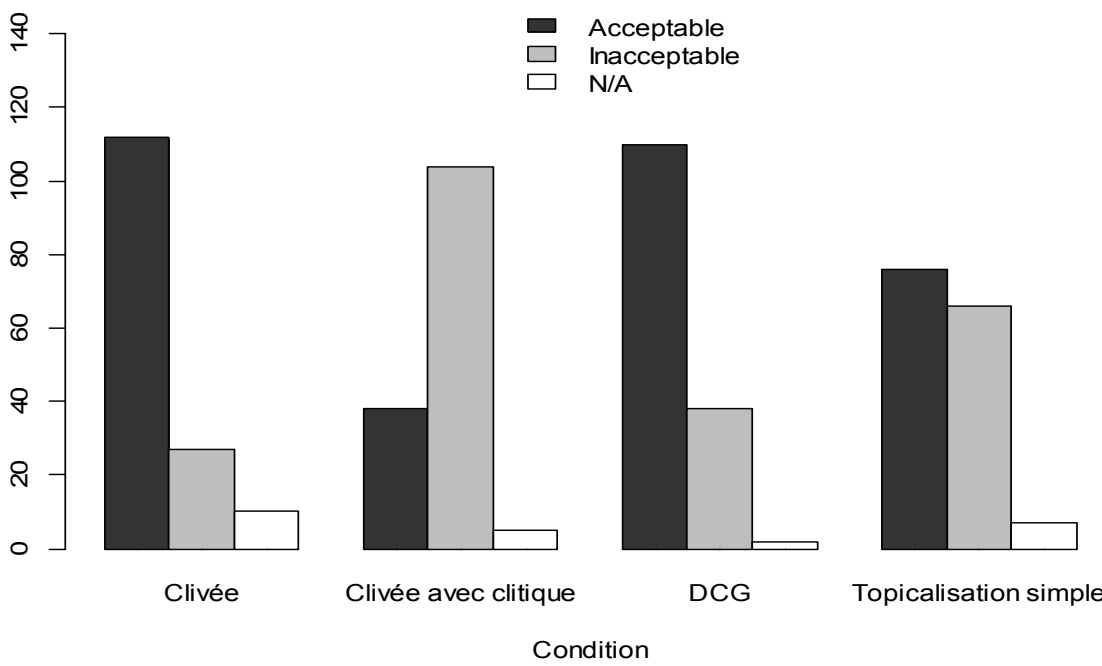

Fig. 1. Nombre et nature des jugements selon les conditions. 
Les jugements ont été divisés en trois catégories: les jugements «acceptable (le participant a jugé l'item acceptable en deux secondes ou moins), les jugements " inacceptable » (le participant a jugé l'item inacceptable en deux secondes ou moins) et les jugements indécis, «N/A » dans le graphique (le participant n'a pas pu juger l'item en deux secondes ou moins).

Pour analyser statistiquement les résultats des différentes conditions, les jugements «acceptable» et «inacceptable» ont été convertis en valeur binaire: «1» pour « acceptable » et « 0 » pour inacceptable. Les jugements «N/A » ont été exclus (10 pour les clivées; 5 pour les clivées avec clitique; 2 pour les DCG; 7 pour les topicalisations simples). Ces données ont ensuite été analysées sur $\mathrm{R}$ à l'aide de tests de régressions logistiques (package «lme4», Bates et al, 2013) avec les paramètres «construction» et « présence du clitique » en tant que facteurs fixes et les facteurs « sujet » et « item » comme facteurs aléatoires (le modèle maximal avec pentes individuelles pour les sujets et les items n'a pas convergé) (Barr et al, 2013). Les tableaux de cette section incluent les résultats pour les facteurs fixes et leur interaction ainsi que le modèle employé.

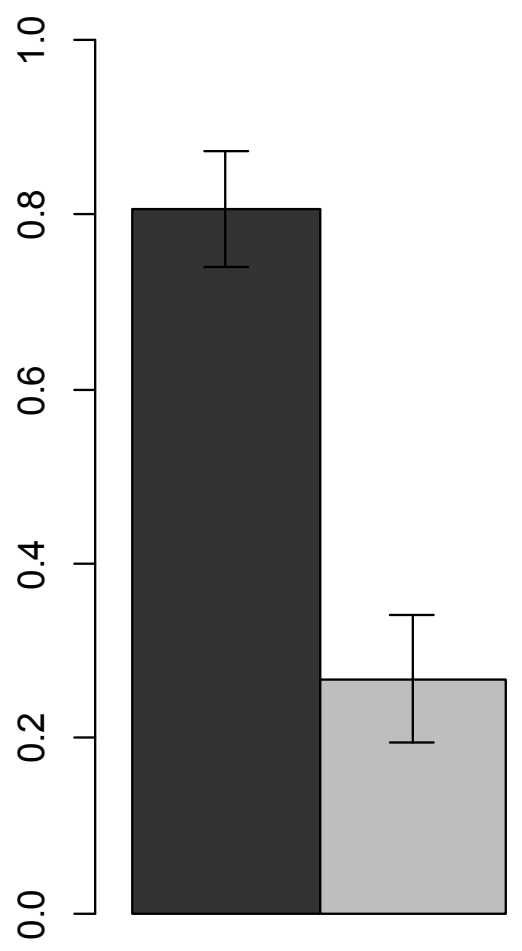

Clivée
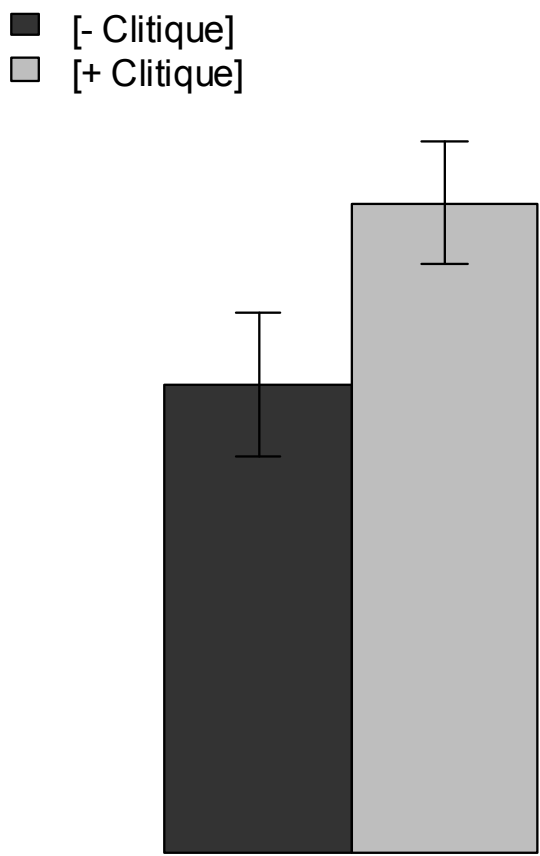

Détachement

\section{Construction}

Fig. 2. Proportion de jugements acceptables selon les conditions.

L'interaction des facteurs « construction » et «présence du clitique » est significative $(\mathrm{p}<.001)$. La présence du clitique améliore significativement l'acceptabilité des dislocations $(p<.001)$ et amoindrie significativement celle des clivées $(p<.001)$. Nous constatons aussi 
un effet significatif du facteur « construction » $(\mathrm{p}<.05)$ et du facteur « clitique » $(\mathrm{p}<.01)$. Une différence significative $(\mathrm{p}<.01)$ existe entre la condition agrammaticale (clivée avec clitique) et la condition «topicalisation simple » et une différence négligeable $(p=.277)$ entre les conditions bien formées (clivée sans clitique et DCG).

Tableau 1. Estimation bêta et valeur-z des facteurs influençant l'acceptabilité.

\begin{tabular}{|l|l|l|}
\hline \multicolumn{2}{|c|}{$\begin{array}{c}\text { glmer(jugement } \sim \text { construction*clitique }+(1 \mid \text { sujet })+(\text { construction*clitique|item }), \\
\text { family=binomial, data) }\end{array}$} \\
\hline & Estimation bêta (Ecart type $)$ & Valeur-z \\
\hline Moyenne générale & $0.58714(0.02745)$ & \\
\hline Construction & $-0.04949(0.02021)$ & -2.5 \\
\hline Présence du clitique & $0.08223(0.02201)$ & 3.74 \\
\hline Construction : Présence du clitique & $0.18529(0.02001)$ & 9.26 \\
\hline
\end{tabular}

\subsubsection{Temps de réaction}

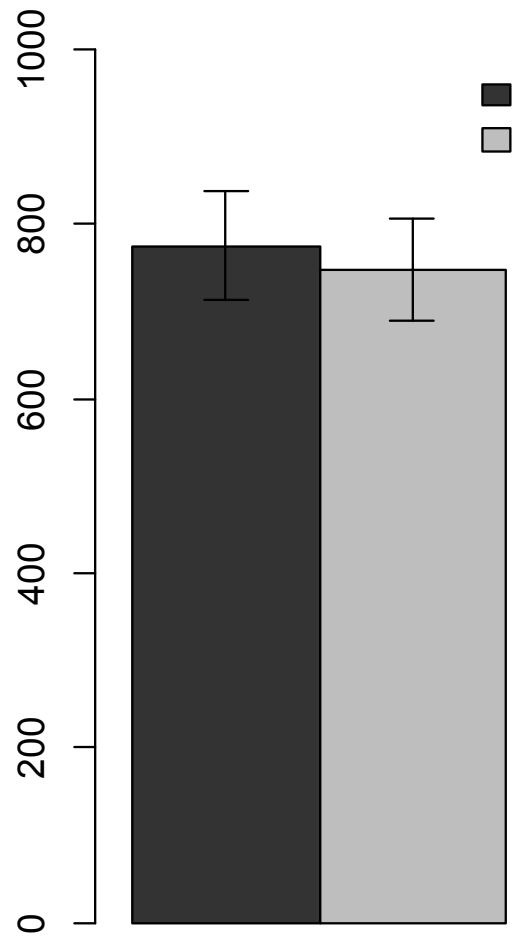

Clivée

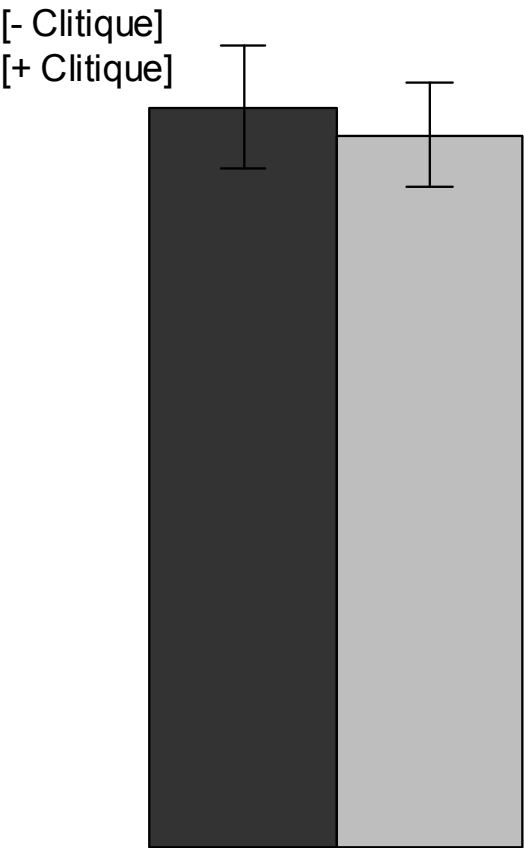

Détachement

\section{Construction}

Fig. 3. Moyenne des temps de réaction selon les conditions. 
Nous avons analysé les temps de réaction avec un modèle linéaire mixte en incluant les facteurs fixes «construction» et «clitique », les facteurs aléatoires «sujet» et «item» ainsi les pentes individuelles pour les items. En ce qui concerne l'analyse des temps de réactions, ni le facteur «construction » $(\mathrm{p}=.124)$ ni le facteur «clitique » $(\mathrm{p}=.172)$ et ni l'interaction des deux facteurs $(p=.697)$ n'ont un effet significatif.

Tableau 2. Estimation bêta et valeur-t des facteurs influençant le temps de réaction.

\begin{tabular}{|l|c|c|}
\hline \multicolumn{2}{|c|}{ lmer(RT $\sim$ construction*clitique $+(1 \mid$ sujet $)+($ construction*clitique|item), data) } \\
\hline & Estimation bêta (Ecart type) & Valeur-t \\
\hline Moyenne générale & $800.005(35.904)$ & \\
\hline Construction & $-35.141(22.076)$ & -1.59 \\
\hline Présence du clitique & $19.828(14.400)$ & 1.38 \\
\hline Construction : Présence du clitique & $-6.107(15.531)$ & -0.39 \\
\hline
\end{tabular}

\section{Interprétation des résultats}

\subsection{Acceptabilité des conditions}

En ce qui concerne l'acceptabilité, les résultats sont cohérents avec notre hypothèse. Les topicalisations simples sont perçues comme étant moins acceptable que les conditions bien formées et dans un contexte approprié (clivées et les DCG). Elles sont toutefois perçues plus acceptables que la condition agrammaticale (clivée avec clitique).

La différence avec la DCG va dans le sens que le contexte présenté (contraste sans mention d'alternative) convient mieux aux détachements de l'objet avec un pronom clitique (DCG) qu'à ceux sans pronom clitique (topicalisation simple). En effet, les DCG sont plus proche du niveau d'acceptabilité établi par notre condition contrôle (clivée sans clitique) par rapport aux topicalisations simples. Dans notre approche à contraintes, nous interprétons cela comme une interaction entre contraintes morphosyntaxiques et discursives. Nous interprétons le niveau d'acceptabilité plus bas des topicalisations simples comme le fait qu'au moins une contrainte de cette construction n'a pas été respectée.

De plus, la différence d'acceptabilité entre les constructions détachées avec et sans clitique et les constructions clivées avec et sans clitique semble indiquer que la contrainte violée pour les topicalisations simples n'est pas de nature catégorique. Si c'était le cas, on s'attendrait à ce que ces deux conditions soient perçues aussi inacceptables l'une que l'autre. Or, la différence d'acceptabilité est ici en faveur des topicalisations simples. Nous interprétons cela comme le fait que la contrainte qui n'est pas respectée est de nature noncatégorique. Ce phénomène expliquerait les effets significatifs observés dans l'analyse de nos résultats.

Toutefois, il est aussi possible que cette différence de moyenne d'acceptabilité soit due à la place du pronom clitique objet dans nos conditions. Sa présence ou son absence est visible en début de phrase pour les détachements à gauche mais plus loin pour les clivées. Lorsque les participants arrivent à l'étape de jugement, il leur est plus facile de se rappeler de la fin de la phrase que du début. Ils ont donc à fournir moins d'efforts pour juger de l'acceptabilité des clivées. Cette interaction entre ordre des mots et cognition pourrait par ailleurs expliquer le caractère non-catégorique de la contrainte de doublage clitique. 


\subsection{Temps de réaction}

L'effet de construction sur les temps de réaction n'est pas forcément cohérent avec notre hypothèse. Dans la mesure où les contraintes non-catégoriques ont un effet moins important sur l'acceptabilité que les contraintes catégoriques, il paraîtrait raisonnable que les participants aient plus de mal à distinguer deux conditions différenciées par une contrainte non-catégorique par rapport à deux conditions différenciées par une contrainte catégorique. Cette difficulté se traduirait par un temps de traitement plus long avant d'établir le jugement d'acceptabilité. Il est compliqué d'interpréter un résultat non-significatif. Il se peut que la contrainte investiguée n'ait pas d'effet sur le temps de réaction. Il se peut également que cet effet demande plus de données (dans notre cas, plus de participants) pour être observable.

\section{Conclusion}

Dans cet article, nous avons fait l'hypothèse que le détachement à gauche de l'objet est affecté par la contrainte de doublage clitique dans un contexte contrastif sans mention d'alternative(s). Nous avons testé cette hypothèse empiriquement via un test de jugements d'acceptabilité accélérés. Les résultats montrent que les détachements à gauche sans doublage clitique (topicalisation simple) sont perçus moins acceptables que les détachements avec doublage clitique (DCG) et une condition de contrôle bien formée (clivée). Ils sont également perçus plus acceptables qu'une condition de contrôle agrammaticale (clivée avec clitique). Ces résultats sont cohérents avec la nature noncatégorique de la contrainte de doublage clitique (Keller et Alexopoulou, 2001 ; Sorace et Keller, 2005).

Ils sont également cohérents avec les descriptions des topicalisations simples (Kerleroux et Marandin, 2001) qui font l'hypothèse que ces constructions soient restreintes à des contextes contrastifs avec mention d'alternative(s). Dans ce cadre, l'effet de la contrainte de doublage clitique pourrait être réduit à l'aide d'un contexte discursif approprié aux détachements de l'objet à gauche sans doublage clitique. Cette hypothèse peut être testée empiriquement via la méthodologie employée dans cet article.

Ces résultats offrent des perspectives intéressantes quant à la description des propriétés pragmatiques des différentes constructions à détachement à gauche. Premièrement, ils posent la question de l'application de la contrainte de doublage clitique à des détachements de compléments non-objet, tel que le complément second de l'exemple (7) (repris cidessous en (20)). L'étude empirique de cette hypothèse permettrait d'étendre la contrainte du doublage clitique à un groupe de constituants plus large que les compléments de l'objet.

20. Marie a réuni les élèves. Aux filles, elle a donné des exercices d'algèbre. Aux garçons, elle a dicté un problème de géométrie.

Deuxièmement, la contrainte inverse s'applique-t-elle aux détachements à gauche avec doublage clitique? En effet, il a été proposé que la mention d'alternative(s) ait un effet négatif sur les DCG (Kerleroux et Marandin, 2001) (21). Dans un contexte contrastif, il y aurait donc une division du travail entre les DCG (contexte sans mention d'alternative) et les topicalisations simples (contexte avec mention d'alternative). La vérification empirique de cette hypothèse permettrait de confirmer la relation entre doublage clitique et contexte discursif pour les détachements de l'objet à gauche.

21. Marie a réuni les élèves. \#Aux filles, elle leur a donné des exercices d'algèbre. Aux garçons, elle leur a dicté un problème de géométrie.

Troisièmement, elle ouvre des perspectives sur le test de la contrainte de doublage clitique dans plusieurs langues. Nous faisons l'hypothèse que la contrainte de doublage clitique pour les objets détachés en français était similaire à la même contrainte pour les objets préverbaux en grec, une langue à l'ordre des mots plus libre que le français. Il serait 
intéressant d'observer si cette contrainte est similaire dans d'autres langues où l'antéposition de l'objet par rapport au verbe est possible.

\section{Références bibliographiques}

Bader, M. et Häussler, J. (2010). Towards a model of grammaticality judgments. Journal of Linguistics, 46, 273-330.

Barr, D.J., Levy, R., Scheepers, C. et Tily, H.J. (2013). Random effects structure for confirmatory hypothesis testing: Keep it maximal. Journal of Memory \& Language, 68, En ligne.

Beyssade, C., Hemforth, B., Marandin, J. M., Portes, C. (2015). Prosodic Realizations of Information Focus in French. Explicit and Implicit Prosody in Sentence Processing, 29-61.

Bates, D. Maechler, M. et Bolker B. (2013). lme4: Linear-mixed Effects Models Using S4 Classes.

Blache, P., Hemforth, B. et Rauzy, S. (2006). Acceptability Prediction by Means of Grammaticality Quantification. Proceedings of COLING-ACL, 57-64.

Brunetti, L., Avanzi, M. et Gendrot, C. (2012). Entre syntaxe, prosodie et discours : les topiques sujets en français parlé. Actes du CMLF 2012, 2041-2054.

Cinque, G. (1990) Types of A bar-dependencies. Cambridge : MIT Press.

De Cat C. (2007). French dislocation : interpretation, syntax, acquisition. Oxford : OUP.

Delais-Roussarie, E., Doetjes, J. et Sleeman, P. (2004). Dislocation. Handbook of French Semantics, 501-529.

Hamlaoui, F. (2009). La focalization à l'interface de la syntaxe et de la phonologie : le cas du français dans une perspective typologique. Paris : Université Sorbonne Nouvelle.

Keller, F. et Alexopoulou, T. (2001). Phonology competes with syntax: Experimental evidence for the interaction of word order and accent placement in the realization of information structure. Cognition, 79, 301-372.

Kerleroux, F. et Marandin, J.-M. (2001). L'ordre des mots. Cahier Jean-Claude Milner, 277-302.

Lambrecht, K. (1994). Information structure and sentence formes: topic, focus and the mental representations of discourse referents. Cambridge : CUP.

Marandin, J.-M., Beyssade, C., Delais-Roussarie, E. et Rialland, A. (2002). Discourse Marking in French : C accents and Discourse Moves. Proceedings of Speech Prosody 2002.

Miller, P. (1992). Clitics and Constituents in Phrase Structure Grammar. New-York : Garland.

Riou, E. et Hemforth, B. (2015). Dislocation clitique de l'objet à gauche en français écrit. Discours, 16, En ligne.

Schneider, W., Eschman, A. et Zucculotto, A. (2012a). E-Prime User's Guide. Pittsburgh : PST Inc.

Schneider, W., Eschman, A. et Zucculotto, A. (2012b). E-Prime Reference Guide Pittsburgh : PST Inc.

Sorace, A. et Keller, F. (2005). Gradience in Linguistic Data. Lingua, 115, 1497-1524.

Zribi-Hertz, A. (1994). The syntax of nominative clitics in standard and advanced French. Paths Towards Universal Grammar: Studies in Honor of Richard S. Kayne, 453-472.

1. Tous nos remerciements à Doriane Gras pour son aide quant à la mise en place de l'expérience sur E-prime.

2. Nous avons choisi d'exclure 3 items de nos résultats, à cause d'un problème de présentation affectant la symétrie entre les conditions. 